\title{
Pressure-Induced Site-Selective Mott Insulator-Metal Transition in $\mathrm{Fe}_{2} \mathrm{O}_{3}$
}

\author{
Eran Greenberg, ${ }^{1, *}$ Ivan Leonov, ${ }^{2,3}$ Samar Layek, ${ }^{1}$ Zuzana Konopkova, ${ }^{4}$ Moshe P. Pasternak, ${ }^{1}$ \\ Leonid Dubrovinsky, ${ }^{5}$ Raymond Jeanloz, ${ }^{6}$ Igor A. Abrikosov, ${ }^{3,7}$ and Gregory Kh. Rozenberg ${ }^{1}$ \\ ${ }^{1}$ School of Physics and Astronomy, Tel Aviv University, 69978, Tel Aviv, Israel \\ ${ }^{2}$ Institute of Metal Physics, Sofia Kovalevskaya Street 18, 620219 Yekaterinburg GSP-170, Russia \\ ${ }^{3}$ Materials Modeling and Development Laboratory, NUST “MISIS," 119049 Moscow, Russia \\ ${ }^{4}$ DESY, HASYLAB, PETRA-III, P02, Notkestraße 85, Building 47c, Hamburg, Germany \\ ${ }^{5}$ Bayerisches Geoinstitut, University of Bayreuth, Bayreuth, Germany \\ ${ }^{6}$ Departments of Earth and Planetary Science and Astronomy, \\ and Miller Institute for Basic Research in Science, University of California, \\ Berkeley, California 94720, USA \\ ${ }^{7}$ Department of Physics, Chemistry and Biology (IFM), Linköping University, \\ SE-581 83 Linköping, Sweden
}

(Received 15 December 2017; revised manuscript received 1 June 2018; published 10 September 2018)

We provide experimental and theoretical evidence for a pressure-induced Mott insulator-metal transition in $\mathrm{Fe}_{2} \mathrm{O}_{3}$ characterized by site-selective delocalization of the electrons. Density functional plus dynamical meanfield theory (DFT + DMFT) calculations, along with Mössbauer spectroscopy, $\mathrm{x}$-ray diffraction, and electrical transport measurements on $\mathrm{Fe}_{2} \mathrm{O}_{3}$ up to $100 \mathrm{GPa}$, reveal this site-selective Mott transition between 50 and $68 \mathrm{GPa}$, such that the metallization can be described by $\left({ }^{\mathrm{VI}} \mathrm{Fe}^{3+\mathrm{HS}}\right)_{2} \mathrm{O}_{3} \quad[R \overline{3} c$ structure $] \underset{50 \mathrm{GPa}}{\longrightarrow}$ $\left({ }^{\mathrm{VIII}} \mathrm{Fe}^{3+\mathrm{HS}} \mathrm{VI}^{\mathrm{Fe}}{ }^{\mathrm{M}}\right) \mathrm{O}_{3}\left[P 2_{1} / n\right.$ structure $] \underset{68 \mathrm{GPa}}{\longrightarrow}\left({ }^{\mathrm{VI}} \mathrm{Fe}^{\mathrm{M}}\right)_{2} \mathrm{O}_{3}[$ Aba2 $/ P P v$ structure $]$. Within the $P 2_{1} / n$ crystal structure, characterized by two distinct coordination sites (VI and VIII), we observe equal abundances of ferric ions $\left(\mathrm{Fe}^{3+}\right)$ and ions having delocalized electrons $\left(\mathrm{Fe}^{\mathrm{M}}\right)$, and only at higher pressures is a fully metallic high-pressure structure obtained, all at room temperature. Thereby, the transition is characterized by delocalization/metallization of the $3 d$ electrons on half the Fe sites, with a site-dependent collapse of local moments. Above approximately $50 \mathrm{GPa}, \mathrm{Fe}_{2} \mathrm{O}_{3}$ is a strongly correlated metal with reduced electron mobility (large band renormalizations) of $m^{*} / m \sim 4$ and 6 near the Fermi level. Importantly, upon decompression, we observe a site-selective (metallic) to conventional Mott insulator phase transition $\left({ }^{\mathrm{VIII}} \mathrm{Fe}^{3+\mathrm{HS}} \mathrm{VI}^{\mathrm{Fe}}{ }^{\mathrm{M}}\right) \mathrm{O}_{3} \underset{50 \mathrm{GPa}}{\longrightarrow}\left({ }^{\mathrm{VIII}} \mathrm{Fe}^{3+\mathrm{HS}} \mathrm{VI} \mathrm{Fe}^{3+\mathrm{HS}}\right) \mathrm{O}_{3}$ within the same $P 2_{1} / n$ structure, indicating a decoupling of the electronic and lattice degrees of freedom. Our results offer a model for understanding insulator-metal transitions in correlated electron materials, showing that the interplay of electronic correlations and crystal structure may result in rather complex behavior of the electronic and magnetic states of such compounds.

DOI: 10.1103/PhysRevX.8.031059

Subject Areas: Condensed Matter Physics, Strongly Correlated Materials

\section{INTRODUCTION}

The insulator-metal transition, induced by pressure, composition, or by other means, represents perhaps the most profound transformation of the chemical bond in

\footnotetext{
*Present address: GSECARS, University of Chicago, Chicago, Illinois 60637, USA.

Corresponding author.

erangre@gmail.com

Published by the American Physical Society under the terms of the Creative Commons Attribution 4.0 International license. Further distribution of this work must maintain attribution to the author(s) and the published article's title, journal citation, and DOI.
}

materials. A specific subset, the Mott transition, is of particular interest because it is thought to depend on electron correlations that are essential to understanding the properties of transition-metal oxides important to fields ranging from materials chemistry to condensed-matter physics and even planetary science. Electronic and magnetic transitions in strongly correlated transition-metal compounds have thus been among the main topics of condensed-matter research over recent decades, being especially relevant to understanding high-temperature superconductivity, as well as heavy-fermion behavior [1-4]. A significant electronic phenomenon in such compounds is the breakdown of $d$ or $f$-electron localization, causing a Mott (Mott-Hubbard) insulator-to-metal transition [1,2]. Such a transition does not 
necessarily imply a rearrangement of atoms, but in fact often exhibits an appreciable collapse in volume [5-8]. The initial concept of Mott is based on the relative importance of kinetic hopping (measured by the bandwidth) and onsite repulsion of electrons. However, in real materials, there exist additional degrees of freedom, which have been shown to result in new scenarios for the Mott transition. For example, orbital degrees of freedom can lead to an orbital-selective Mott transition $[9,10]$. In addition, it was proposed that a change of the crystal-field splitting (rather than the bandwidth), or a decrease of the effective interaction strength (given by the Hubbard $U$ parameter) caused by a high spin-low spin (HS-LS) crossover, can drive a Mott transition [7,11-13]. As a result, the Mott transition in principle involves a simultaneous insulator-metal transition, magnetic moment collapse (change of the local spin state), and volume collapse.

Nevertheless, the abovementioned scenarios of Mott transitions do not explain the experimentally observed details of electronic and structural transformations, e.g., in many iron-bearing materials. In particular, recent experimental studies of ferric and ferrous spinels [14-17] and Fe-bearing bridgmanite $\left(\mathrm{MgSiO}_{3}\right.$-peroskite) $[18,19]$ reveal a complex coexistence of local $\mathrm{Fe}^{3+}$ high- and low-spin states under pressure. In spite of the appreciable $3 d$ band broadening from various lattice responses that contribute to the unit-cell reduction, upon compression to a pressure of a megabar, all of these compounds continue to have concurrent resilient magnetic (high-spin) and nonmetallic ground-state features of strong electron correlations. Moreover, a similar behavior has been recently discussed in the case of rare-earth nickelates [20,21], where theoretical calculations have suggested formation of a site-selective Mott phase. However, the latter remains electrically insulating, while exhibiting formation of site-selective local moments and Ni- $d-\mathrm{O}-p$ singlet states.

This complex electronic state characterized by the coexistence of high-spin (HS) and low-spin (LS) states has also been observed in an archetypal $3 d$ Mott insulator (MI) $\mathrm{Fe}_{2} \mathrm{O}_{3}$ hematite [22-28]. However, in contrast to the above-mentioned systems, in $\mathrm{Fe}_{2} \mathrm{O}_{3}$, a partial HS-LS transition coincides with a Mott insulator-to-metal transition [23], suggesting a breakdown of $3 d$-electron localization. In this respect, the high-pressure behavior of $\mathrm{Fe}_{2} \mathrm{O}_{3}$ is of particular interest as possibly documenting a novel mechanism for electronic transitions.

$\mathrm{Fe}_{2} \mathrm{O}_{3}$ crystallizes in a corundum-type structure (space group $R \overline{3} c$, Néel temperature $T_{\mathrm{N}}=956 \mathrm{~K}$ ) with one type of $\mathrm{FeO}_{6}$ octahedron (slightly distorted). Photoemission spectroscopy shows that $\mathrm{Fe}_{2} \mathrm{O}_{3}$ is a Mott insulator with a large energy gap of approximately $2.5 \mathrm{eV}$ [29]. Upon compression, $\mathrm{Fe}_{2} \mathrm{O}_{3}$ is known to undergo a first-order phase transition above $50 \mathrm{GPa}$, which is accompanied by a collapse of the lattice volume by about $10 \%$ [22,23,30-33]. The phase transition was associated with a "partial" transition to the low-spin $\mathrm{Fe}^{3+}$ state [22,24-27]. However, subsequent Mössbauer studies indicated that, at $P>80 \mathrm{GPa}$, only a nonmagnetic phase exists, without any sign of magnetic moments down to $4 \mathrm{~K}$ [23]. Furthermore, although early electrical transport measurements [34] claimed that-despite the sharp drop in resistance coinciding with the structural transition- $\mathrm{Fe}_{2} \mathrm{O}_{3}$ remains semiconducting, a later study [23] showed that, in fact, there is a sharp insulator-metal transition at a substantially lower pressure of $50 \mathrm{GPa}$. The latter result seemingly contradicts of the high-spin state at pressures far above this transition, since the high-spin state would not be expected to exhibit the electrical conductivity of a metal.

The crystal structure of the high-pressure phase of $\mathrm{Fe}_{2} \mathrm{O}_{3}$ observed above $50 \mathrm{GPa}$ has been assigned either to an orthorhombic perovskite $[22,31,35]$ or a $\mathrm{Rh}_{2} \mathrm{O}_{3}$-II-type $[23,32]$ structure in early studies. Only recently, based on single-crystal diffraction studies $[28,36]$, has the lattice structure been defined as a double-perovskite (DPv) phase. It has been proposed [32,37] that this structural transition drives the electronic and magnetic transformation. Furthermore, it was reported [37] that the system reverses, as a function of time, from the LS to the HS state within the HP crystal structure. However, in more recent studies (e.g., Sanson et al. [38]), the opposite was advocated, namely, that the electronic transition drives the structural transition. In addition, recent density-functional theory + dynamical mean-field theory (DFT + DMFT [39]) calculations predict that the electronic transition occurs within the hematite phase [40], i.e., prior to the structural transition, at a high compression of $V<0.8 V_{0}$ ( $V_{0}$ is the equilibrium unit-cell volume), which, according to an experimental equation of state (EOS), e.g., Refs. [31,32], corresponds to $P>70 \mathrm{GPa}$. We note that, in Ref. [40], the structural complexity of $\mathrm{Fe}_{2} \mathrm{O}_{3}$ near the phase transition has not been considered; i.e., the interplay between correlated electrons and the lattice structure was not studied. Thus, despite significant efforts on this outwardly simple system, the evolution with pressure of the electronic and crystallographic structure of $\mathrm{Fe}_{2} \mathrm{O}_{3}$ and the mechanism of its electronic transition remain unresolved. Current theoretical models [40] do not explain the existing experimental results [22-27,37].

In the present work, we employ the DFT + DMFT approach to explore the electronic structure, local magnetic state of $\mathrm{Fe}^{3+}$ ions, and phase stability of paramagnetic $\mathrm{Fe}_{2} \mathrm{O}_{3}$ at high pressure, using a fully charge self-consistent DFT + DMFT method [6,41] implemented with planewave pseudopotentials [42-44]. We combine our theoretical results with Mössbauer spectroscopy (MS), electrical transport, and x-ray diffraction (XRD) studies of $\mathrm{Fe}_{2} \mathrm{O}_{3}$ to pressures of about $100 \mathrm{GPa}$, upon compression and decompression. Our study reveals a site-selective Mott insulator-metal transition in $\mathrm{Fe}_{2} \mathrm{O}_{3}$ characterized by delocalization and, hence, metallization of the $\mathrm{Fe} 3 d$ electrons on only half of the $\mathrm{Fe}$ sites within the crystallographic unit 
cell: a transition accompanied by collapse of the local moments on the same octahedral Fe sites of the distorted $\mathrm{DPv}$ structure. This behavior clearly distinguishes the transformation in $\mathrm{Fe}_{2} \mathrm{O}_{3}$ from conventional and orbitalselective Mott metal-insulator transitions. The $\mathrm{DPv}$ phase of $\mathrm{Fe}_{2} \mathrm{O}_{3}$ is a strongly correlated metal with reduced mobility (high effective mass, $m^{*}$ ) of electrons near the Fermi energy, $m^{*} / m \sim 4$ to 6 , and upon decompression it reverts back to a conventional Mott insulator ( $m$ is the normal mass of the electron). Our work highlights the interplay between electronic and crystalline structures, and not only addresses a long-standing controversy regarding the high-pressure behavior of $\mathrm{Fe}_{2} \mathrm{O}_{3}$, but also suggests that site-selective Mott transitions can occur more generally for transition-metal compounds.

\section{METHODS AND MATERIALS}

We employ a state-of-the-art DFT + DMFT approach, fully self-consistent in charge density, with the average Coulomb interaction $U=6 \mathrm{eV}$ and Hund's exchange $J=0.86 \mathrm{eV}$ for the $\mathrm{Fe} 3 d$ shell [40] to explore the electronic structure, $\mathrm{Fe}^{3+}$ magnetic state, and lattice stability of $\mathrm{Fe}_{2} \mathrm{O}_{3}$. We use this theory to systematically study the high-pressure phase equilibrium of the corundum $(R \overline{3} c), \mathrm{DPv}\left(P 2_{1} / n\right), \mathrm{Rh}_{2} \mathrm{O}_{3}$-II-type $(P b c n)$, orthorhombic $\mathrm{Aba2}$, and postperovskite (PPv) $\left(\mathrm{CaIrO}_{3}\right.$-type, $\left.\mathrm{Cmcm}\right)$ crystal structures of $\mathrm{Fe}_{2} \mathrm{O}_{3}$. To solve the realistic manybody problem, we use the continuous-time hybridizationexpansion (segment) quantum Monte Carlo algorithm [45]. We employ the fully localized double-counting correction, evaluated from the self-consistently determined local occupancies, to account for the electronic interactions already described by DFT. Further technical details about the methods used can be found in the Supplemental Material [46].

All measurements for the present study were performed on $\mathrm{Fe}_{2} \mathrm{O}_{3}$ powder ( $99.5 \%$ pure) commercially available from Riedel-de Haën. For Mössbauer studies, 30\% enriched ${ }^{57} \mathrm{Fe}_{2} \mathrm{O}_{3}$ was used. Custom diamond anvil cells (DACs) were used to induce high pressure, with neon as a pressure-transmitting medium. Pressure was determined using the ruby R1 fluorescence line as a pressure marker, and the Ne unit-cell volume in the case of $\mathrm{x}$-ray diffraction studies.

${ }^{57} \mathrm{Fe}$ Mössbauer studies were performed using a $10 \mathrm{mCi}$ ${ }^{57} \mathrm{Co}(\mathrm{Rh})$ point source in a variable temperature $(5-300 \mathrm{~K})$ cryostat. Powder XRD experiments were performed at the Extreme Conditions Beamline (ECB) P02.2 at PETRA III, Hamburg, Germany. Details of the single-crystal diffraction experiments are given in Refs. [28,36]. Electrical resistance measurements were performed as a function of pressure and temperature using a standard four-probe method in a custom-made cryostat.

\section{RESULTS}

\section{A. DFT + DMFT calculations}

In Fig. 1, we show the pressure dependence of the unitcell volume and phase equilibria of $\mathrm{Fe}_{2} \mathrm{O}_{3}$ obtained in our calculations together with the experimental data from this study, as well as taken from the literature [28]. As a starting point, let us consider the electronic and structural properties of the low-pressure $R \overline{3} c$ phase calculated in the paramagnetic state using DFT + DMFT. In Fig. 2, we present our results for the total energy and local moments of the $R \overline{3} c$ phase of $\mathrm{Fe}_{2} \mathrm{O}_{3}$ under pressure (see Supplemental Material [46] pp. 4-7 for further details). In agreement with previous studies [40], we obtain a Mott insulating solution with a relatively large $d$ - $d$ energy gap of about $2.5 \mathrm{eV}$. The calculated equilibrium lattice constant 5.61 a.u. (lattice volume $V=107.3 \AA^{3}$ ) and bulk modulus of approximately $187 \mathrm{GPa}$ are in good agreement with the XRD measurements (see Supplemental Table SIII [46]). Our result for the local magnetic moments is approximately $4.76 \mu_{\mathrm{B}}$, documenting that, at ambient pressure, the $\mathrm{Fe}^{3+}$ ions are in a high-spin state $(S=5 / 2)$ with localized $3 d$ electrons. Under pressure, the energy gap is found to gradually decrease, resulting in a Mott insulator-to-metal phase transition (within the $R \overline{3} c$ structure) below $V \sim 0.74 V_{0}$, at about $72 \mathrm{GPa}$. The phase transition is

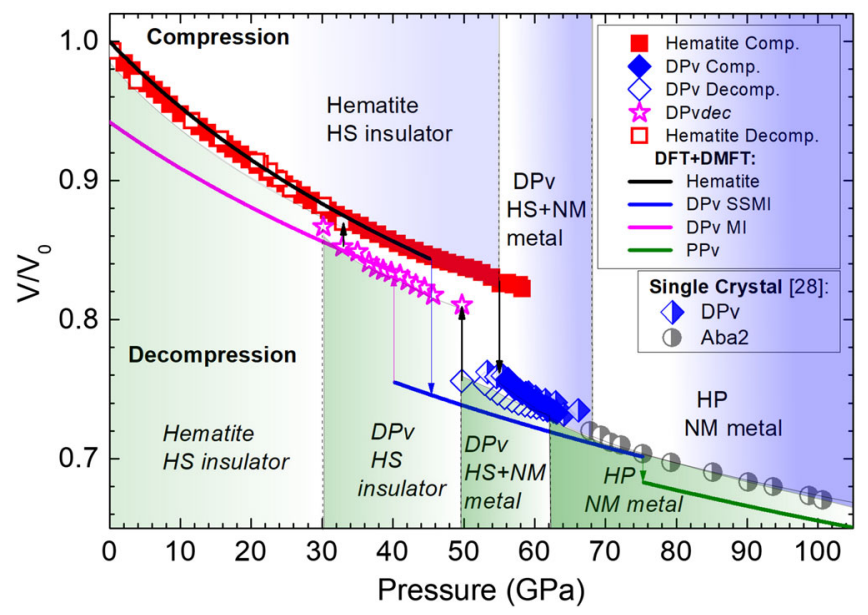

FIG. 1. Pressure dependence of the unit-cell volume of $\mathrm{Fe}_{2} \mathrm{O}_{3}$ at room temperature $\left[V_{0}\right.$ is the unit-cell volume of the hematite phase at 1 bar and $300 \mathrm{~K}$ (for details, see Table S-III [46]); lattice volume is normalized to 2 formula units of $\mathrm{Fe}_{2} \mathrm{O}_{3}$, and volume error bars are within the symbol sizes]. Solid and open symbols indicate compression and decompression (powder), respectively, and half-filled symbols correspond to the single-crystal compression experiments (DPv and Aba2 structures) [28]. Results of first-principles (DFT + DMFT) calculations for the hematite, double-perovskite (DPv) and postperovskite $\left(\mathrm{PPv}, \mathrm{CaIrO}_{3}\right.$ structure) phases are shown by solid lines. Additional abbreviations are defined as follows: double-perovskite phase formed on decompression $\left(\mathrm{DPv}_{\mathrm{dec}}\right)$, high-pressure phase $(\mathrm{HP})$, high-spin (HS), Mott insulator (MI), nonmagnetic (NM). 

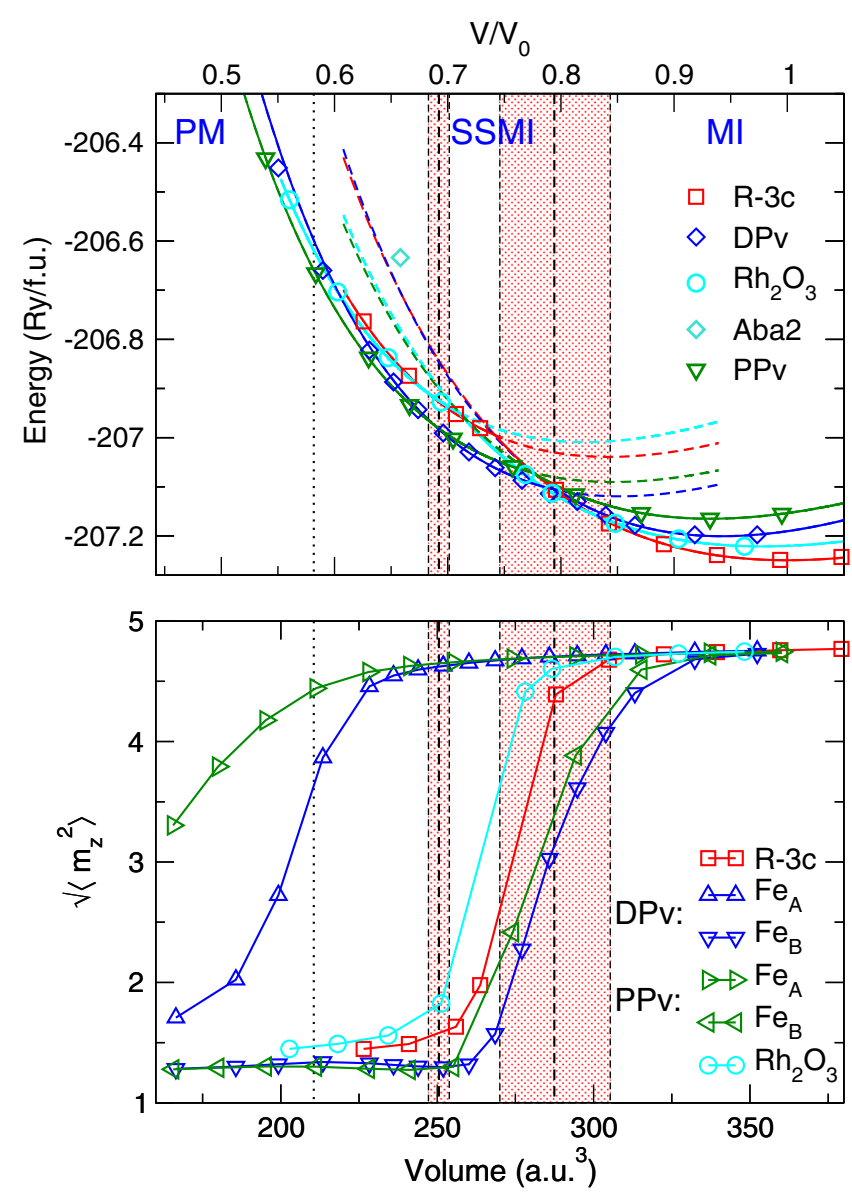

FIG. 2. Total energy and local magnetic moment $\sqrt{\left\langle\hat{m}_{z}^{2}\right\rangle}$ of paramagnetic $\mathrm{Fe}_{2} \mathrm{O}_{3}$ calculated by DFT + DMFT for different lattice volumes at $T=1160 \mathrm{~K}$, where MI stands for Mott insulator, SSMI for site-selective MI, and PM for paramagnetic metal. Above $45 \mathrm{GPa}$, the hematite-structured $(R \overline{3} c)$ MI phase transforms to the double-perovskite (DPv) SSMI phase, which, above $75 \mathrm{GPa}$, further collapses to the postperovskite (PPv) PM phase. The corresponding phase transitions are shown by vertical dashed lines. Our results for the lattice volume collapse are marked by red shading.

associated with a spin-state transition, from HS to LS, with all the $\mathrm{Fe}^{3+}$ ions collapsed to a LS state (with a local moment of approximately $1.5 \mu_{\mathrm{B}}$ ) [40]. We note, however, that these calculations predict the HS-LS and Mott insulator-to-metal transitions, within the $R \overline{3} c$ phase of $\mathrm{Fe}_{2} \mathrm{O}_{3}$, to occur upon compression above $72 \mathrm{GPa}$, i.e., at substantially higher compression (by about 40\%) than $50 \mathrm{GPa}$ found in the previous experimental studies [22-25,27,28,37] and confirmed in our present experiments (see Supplemental Material [46] p. 5).

Along with the $R \overline{3} c$ phase, we have calculated the electronic and structural properties of the $\mathrm{DPv}$ and $\mathrm{Rh}_{2} \mathrm{O}_{3}$-II-type phases observed experimentally above $50 \mathrm{GPa}$, using structural data from $[28,32,36]$ combined with the present experimental data. We calculate the electronic properties of $\mathrm{DPv} \mathrm{Fe}_{2} \mathrm{O}_{3}$ using the monoclinic
$P 2_{1} / n$ symmetry and crystal structure parameters as obtained by single-crystal XRD at about $54 \mathrm{GPa}$ [36]. The DPv lattice has the general formula $A_{2} B^{\prime} B^{\prime \prime} \mathrm{O}_{6}$, with two structurally distinct $\mathrm{Fe} A$ and $B$ positions due to differing oxygen surroundings: A sublattice of octahedral Fe $B$ sites build a three-dimensional network of tilted corner-sharing $B^{\prime} \mathrm{O}_{6}$ and $B^{\prime \prime} \mathrm{O}_{6}$ octahedra, with $\mathrm{Fe} A$ cations located in bicapped trigonal-prismatic voids (eight nearest neighbors). Therefore, we use a cluster expansion of the DFT + DMFT approach in order to treat correlations in the $\mathrm{Fe} 3 d$ bands of the structurally distinct $\mathrm{Fe} A$ and $\mathrm{Fe} B$ sites. For the $\mathrm{Rh}_{2} \mathrm{O}_{3}$-II phase of $\mathrm{Fe}_{2} \mathrm{O}_{3}$, we used the crystal structure parameters determined at approximately $41 \mathrm{GPa}$ in Ref. [28].

In agreement with experiment, the $R \overline{3} c$ phase is found to be energetically favorable at low compression, with a total energy difference of about $0.67 \mathrm{eV} / \mathrm{f}$.u. (formula unit) between the $R \overline{3} c$ and $\mathrm{DPv}$ structures. While the total energy difference between the $R \overline{3} c$ and $\mathrm{Rh}_{2} \mathrm{O}_{3}$-II phases is approximately $0.35 \mathrm{eV} / \mathrm{f}$.u., the $\mathrm{Rh}_{2} \mathrm{O}_{3}$-II structure remains thermodynamically unstable, with a total energy difference of $\Delta E \sim 42 \mathrm{meV} /$ f.u. (with respect to the $\mathrm{DPv}$ phase) for $0.84 V_{0}$, and $\Delta E \sim 420 \mathrm{meV} /$ f.u. upon compression to $V \sim 0.75 V_{0}$. This indicates that the $\mathrm{Rh}_{2} \mathrm{O}_{3}$-IItype phase is metastable at high pressures, in agreement with recent experiments [28]. Most importantly, our calculations reveal a phase transition from a corundum to a $\mathrm{DPv}$ crystal structure upon compression to about $0.84 \mathrm{~V} / V_{0}$, above $45 \mathrm{GPa}$. The phase transition is accompanied by a collapse of the lattice volume by $11.6 \%$. The calculated bulk modulus of the DPv phase is $259 \mathrm{GPa}$, which is substantially larger than that obtained for the HS state of the $R \overline{3} c$ phase (187 GPa). Our theoretical results are in overall quantitative agreement with the results of XRD experiments, where upon compression above $50 \mathrm{GPa}$, the corundum $R \overline{3} c$ phase undergoes a structural transition to the DPv phase (see Sec. III C and Fig. 1).

Most interestingly, the $R \overline{3} c$-to-DPv phase transition is accompanied by a site-selective collapse of local moments (see Fig. 2): The local magnetic moment at the Fe $A$ sites is about $4.63 \mu_{\mathrm{B}}$, which differs substantially from the magnetic moment at the $B$ sites of $0.89 \mu_{\mathrm{B}}$. Thus, our calculations show that, in the $\mathrm{DPv}$ phase, the cations occupying the octahedral $\mathrm{Fe} B$ sites are in a low-spin state $(S=1 / 2)$, while those in the Fe $A$ sites remain high-spin $(S=5 / 2)$. Moreover, our results for the spectral function (Fig. 3) reveal the existence of a site-selective Mott insulator (SSMI) phase, in which the $3 d$ electrons on only half of the Fe sites (octahedral $B$ sites) are metallic, while the $A$ sites remain insulating. It is noteworthy that this site-selective behavior is in qualitative difference from a site-selective Mott-insulating state that has recently been discussed in the rare-earth nickelates [20,21]. In particular, the nickelates were shown to remain insulating while exhibiting formation of site-selective local moments and 


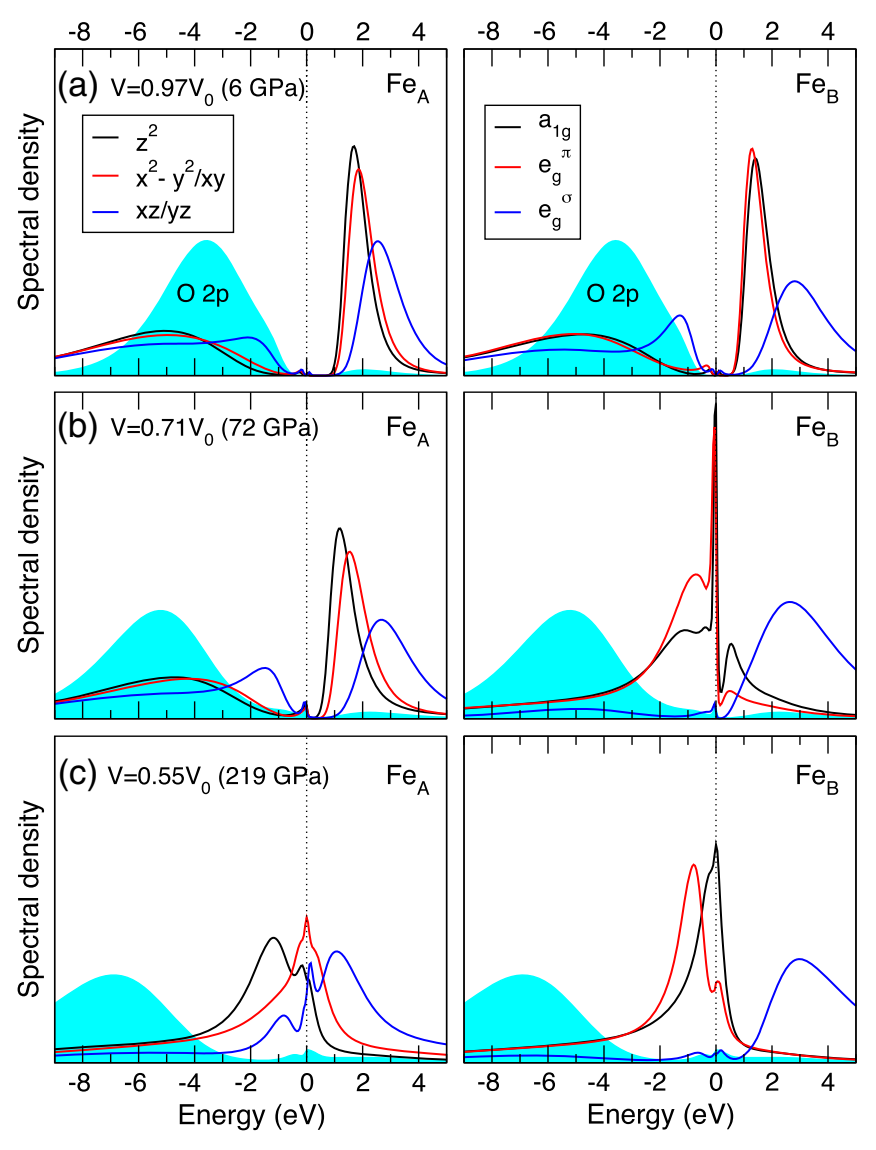

FIG. 3. Spectral function for paramagnetic $\mathrm{DPv} \mathrm{Fe}_{2} \mathrm{O}_{3}$ calculated by $\mathrm{DFT}+\mathrm{DMFT}$ at temperature $T=390 \mathrm{~K}$ and various lattice volumes, based on crystal structure parameters taken from the x-ray diffraction results at approximately $54 \mathrm{GPa}$. Fe $3 d$ (black, red, and blue curves) and O $2 p$ (blue-shaded area) orbitals are shown relative to the Fermi energy $\left(E_{F}=0\right)$. DFT + DMFT results reveal the existence of a site-selective Mott phase at $V=$ $0.71 V_{0}(\mathrm{~b})$, in which the $3 d$ electrons of only half of the Fe sites (right: $B$ sites) are metallic, while the other Fe (left: $A$ sites) remain insulating. For $V=0.97 V_{0}(6 \mathrm{GPa})$, we obtain a conventional Mott insulating phase in which both the $\mathrm{Fe} A$ and $B$ sublattices are insulating (a). Under a high pressure of approximately $219 \mathrm{GPa}\left(0.55 V_{0}\right)$, DFT + DMFT give a metallic solution for both the Fe $A$ and $B$ sites (c).

Ni- $d-\mathrm{O}-p$ singlet states. We conclude that $\mathrm{Fe}_{2} \mathrm{O}_{3}$ undergoes a MI-to-SSMI phase transition upon compression above $50 \mathrm{GPa}$; the Mott-insulator transition goes along with the $R \overline{3} c$-to-DPv structural transformation and is associated with a site-selective collapse of local moments. Thereby, our results reveal a novel type of pressure-induced insulator-metal transition-a site-selective Mott insulator-metal transition, characterized by site-selective delocalization of the $\mathrm{Fe} 3 d$ electrons.

Moreover, our results exhibit a site-selective redistribution of the Fe $3 d$ charges between the $t_{2 \mathrm{~g}}$ and $e_{\mathrm{g}}$ orbitals associated with the HS-LS transition, which is found to appear in the SSMI phase of $\mathrm{Fe}_{2} \mathrm{O}_{3}$ (see Fig. 3). It is accompanied by a collapse of local magnetic moments of the Fe $3 d$ electrons in the octahedral Fe $B$ lattice of the DPv phase. Our results for the $\mathrm{DPv}$ phase show that the octahedral Fe $B t_{2 \mathrm{~g}}$ orbital occupations gradually increase upon compression to $V \sim 0.75 V_{0}$. In fact, the $a_{1 \mathrm{~g}}$ orbital occupancy is about 0.75 , the $e_{\mathrm{g}}^{\pi}$ occupation of 0.85 , upon compression above $50 \mathrm{GPa}$, while the $\mathrm{Fe} B e_{\mathrm{g}}^{\sigma}$ orbitals are strongly depopulated (their occupation is below 0.2) (Fig. 4). We predict that a HS-LS transition within the prismatic $\mathrm{Fe} A$ sublattice should occur at a significantly higher compression of approximately $0.6 V_{0}$, above $190 \mathrm{GPa}$.

The Fe $B a_{1 \mathrm{~g}}$ and $e_{\mathrm{g}}^{\pi}$ orbitals show a sharp quasiparticle peak at the Fermi level, which is associated with a pronounced (orbital-selective) enhancement of the effective electron mass, $m^{*} / m$. In fact, we estimate $m^{*} / m \sim 6$ for the Fe $B \quad a_{1 \mathrm{~g}}$ and approximately 4 for the $e_{\mathrm{g}}^{\pi}$ orbitals at a temperature of approximately $390 \mathrm{~K}$. In contrast to that, the Fe $B e_{\mathrm{g}}^{\sigma}$ orbitals remain insulating. Furthermore, our results for the spin-spin correlation function $\chi(\tau)=\left\langle\mathrm{m}_{\mathrm{z}}(\tau) \mathrm{m}_{\mathrm{z}}(0)\right\rangle$ (Fig. 5) show that the $3 d$ electrons on the Fe $A$ ions are localized to form fluctuating moments $[\chi(\tau)$ is seen to be almost constant and close to unity]. In contrast, the $3 d$ electrons on the $\mathrm{Fe} B$ ions exhibit a rather itinerant magnetic behavior for the $a_{1 \mathrm{~g}}$ and $e_{\mathrm{g}}^{\pi}$ orbitals, implying a localized-to-site-selective itinerant moment transition in $\mathrm{Fe}_{2} \mathrm{O}_{3}$ under pressure.

Upon lattice expansion of the $\mathrm{DPv}$ structure to $V / V_{0} \sim 0.75$, below $40 \mathrm{GPa}$, our calculations suggest an isostructural phase transition into a conventional Mott insulating state coinciding with a significant volume change of 9\% (see Figs. 1 and 3, upper panel). This implies a transition between site-selective and conventional Mott phases within the DPv structure upon decompression

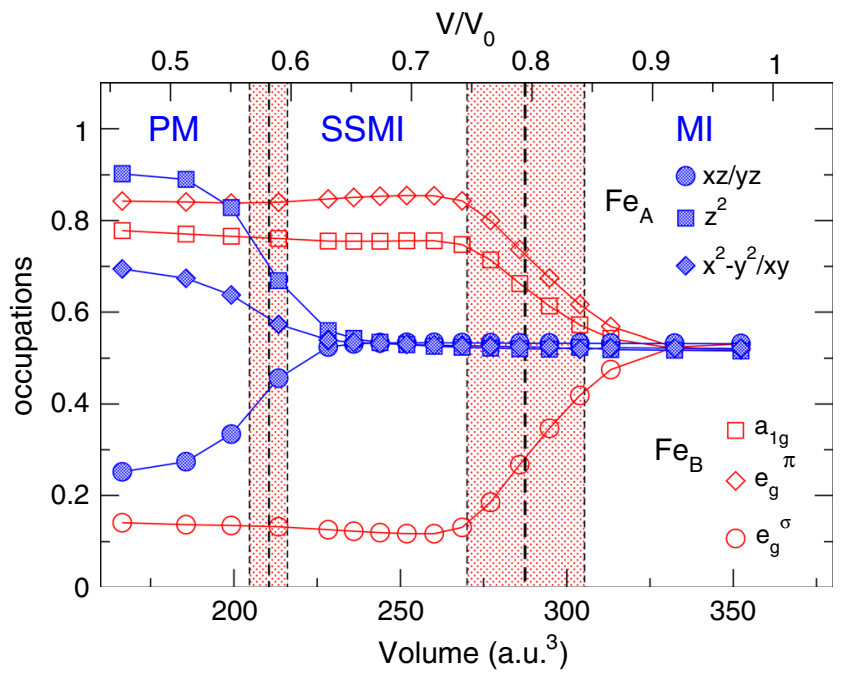

FIG. 4. Partial Fe $3 d$ occupations of paramagnetic DPv $\mathrm{Fe}_{2} \mathrm{O}_{3}$ calculated by DFT + DMFT as a function of lattice volume. 


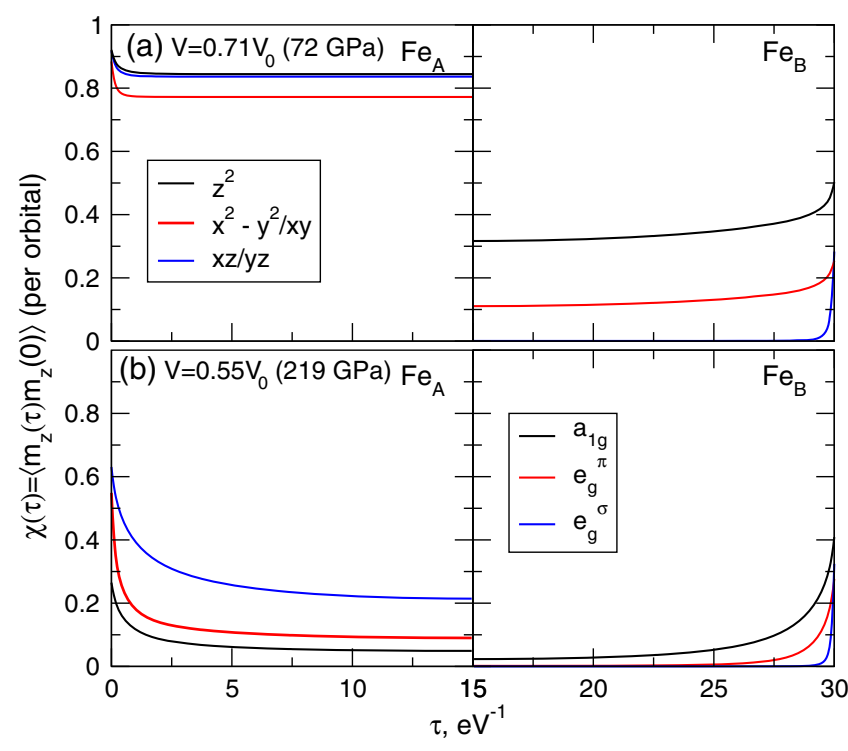

FIG. 5. Local spin-spin correlation function $\chi(\tau)=$ $\left\langle\mathrm{m}_{\mathrm{z}}(\tau) \mathrm{m}_{\mathrm{z}}(0)\right\rangle$ calculated by DFT + DMFT for paramagnetic $\mathrm{DPv} \mathrm{Fe}_{2} \mathrm{O}_{3}$.

and recompression, suggesting the possibility of experimentally observing a decoupling of electronic and lattice degrees of freedom in $\mathrm{Fe}_{2} \mathrm{O}_{3}$.

How stable is the site-selective Mott insulator phase in the DPv phase of $\mathrm{Fe}_{2} \mathrm{O}_{3}$ ? Our calculations show that, upon compression above $192 \mathrm{GPa}, V \sim 0.6 V_{0}$, there is a HS-LS phase transition of the prismatic Fe $A$ electrons, accompanied by a $5 \%$ collapse of the lattice volume. The latter transition is associated with formation of a conventional metallic state of the $\mathrm{Fe}_{2} \mathrm{O}_{3} \mathrm{DPv}$ phase, which is clearly seen from our results for the spectral function (see Fig. 3, bottom). However, the transition pressure is well above the thermodynamic stability range of the $\mathrm{Fe}_{2} \mathrm{O}_{3} \mathrm{DPv}$ phase (Fig. 1).

We therefore explore further the phase stability of paramagnetic $\mathrm{Fe}_{2} \mathrm{O}_{3}$ at high pressures, and examine two candidates for the high-pressure crystal structure proposed from experiment [28]: the $\mathrm{Aba} 2$ and $\mathrm{CaIrO}_{3}$-type $\mathrm{PPv}$ crystal structures. The former has been reported to have an orthorhombic space group. However, it is metastable with a stability range limited to low temperatures, and it is found to transform into the $\mathrm{PPv}$ structure upon annealing at high temperatures [28]. Our total-energy calculations for the $A b a 2$ crystal structure confirm its thermodynamic instability (see Fig. 2), implying that $A b a 2 \mathrm{Fe}_{2} \mathrm{O}_{3}$ is indeed metastable at high pressures. In fact, we have checked the conclusion with different computational parameters, e.g., $U$ and $J$, and found that the obtained result was robust. However, we cannot exclude a possible stabilization of the $A b a 2$ phase due to nonlocal correlation effects, e.g., due to dimerization of $\mathrm{Fe}^{3+}$; the latter are not taken into account in our single-site DFT + DMFT calculations. At the same time, our results for the $\mathrm{PPv}$ phase suggest a structural phase transition from the DPv to PPv phase above $75 \mathrm{GPa}$ (Fig. 1), in quantitative agreement with available experiments [28]. The phase transition is accompanied by about $2.6 \%$ collapse of the lattice volume and is associated with formation of a metallic state (both crystallographic Fe sites are metallic). Interestingly, our results for the local moments reveal site-selective local moment behavior for the PPv phase similar to that found in the DPv phase. The HS-LS transition of the prismatic Fe $A$ electrons in $\mathrm{PPv}$ $\mathrm{Fe}_{2} \mathrm{O}_{3}$ is found to occur at substantially higher compression, $V \sim 0.45 V_{0}$, or about $400 \mathrm{GPa}$. This suggests that metallization of $\mathrm{Fe} A$ sites in the $\mathrm{PPv}$ phase is not related to a spin crossover.

Overall, our results for the phase stability of $\mathrm{Fe}_{2} \mathrm{O}_{3}$ under pressure demonstrate that $\mathrm{Fe}_{2} \mathrm{O}_{3}$ undergoes a phase transition from the low-pressure corundum to the high-pressure $\mathrm{DPv}$ structure, which is accompanied by a transition from a Mott insulator to a site-selective Mott metal insulator at about $50 \mathrm{GPa}$. The behavior of $\mathrm{Fe}_{2} \mathrm{O}_{3}$ at even higher pressure deserves further experimental investigation.

To verify our most important theoretical result on the observation of the site-selective Mott insulator phase in the high-pressure $\mathrm{DPv}$ structure, we further complement our computations of the electronic structure and spin state of $\mathrm{Fe}_{2} \mathrm{O}_{3}$ with results of combined Mössbauer spectroscopy, electrical transport, and XRD studies to pressures of about $100 \mathrm{GPa}$, upon compression and decompression.

\section{B. Mössbauer spectroscopy}

Mössbauer spectra of $\mathrm{Fe}_{2} \mathrm{O}_{3}$, characteristic of various pressure ranges and recorded at room and low temperatures, are shown in Figs. 6(a), 6(b), and 6(d). In agreement with previous publications [22-25], the only observed spectral component upon compression is that of the high-spin state up to nearly $48 \mathrm{GPa}$ [hematite phase in Fig. 6(a)]. At $P \geq 48 \mathrm{GPa}$, two new equally abundant components emerge: a nonmagnetic quadrupole-split component $(\mathrm{nm})$, with no sign of magnetic correlations down to $8 \mathrm{~K}$ [Fig. 6(b)], and a magnetically split component $(\mathrm{m})$ characterized by a significantly reduced hyperfine field $H_{h f}$ [Supplemental Figure S1(a) [46] ]. Taking into account the reported double-perovskite-type structure in this pressure range [36], along with computation results, the two components are designated $\mathrm{DPv}_{n m}$ and $\mathrm{DPv}_{m}$, respectively. At $P \geq 56 \mathrm{GPa}$, the only spectral components are $\mathrm{DPv}_{n m}$ and $\mathrm{DPv}_{m}$ with equal abundances, until at $P \geq 62 \mathrm{GPa}$ the abundance of a nonmagnetic component starts to increase [Fig. 7(a)] to the point that, above $75 \mathrm{GPa}$, the Mössbauer spectra show a single, quadrupole-split component, designated as the high-pressure (HP) state [Fig. 6(d)]. The lack of any signs of magnetic correlations on Mössbauer timescales (approximately $10^{-7} \mathrm{~s}$ ) down to $4 \mathrm{~K}$ [Fig. 6(d)] prompted us to designate this single HP component as a nonmagnetic state. 


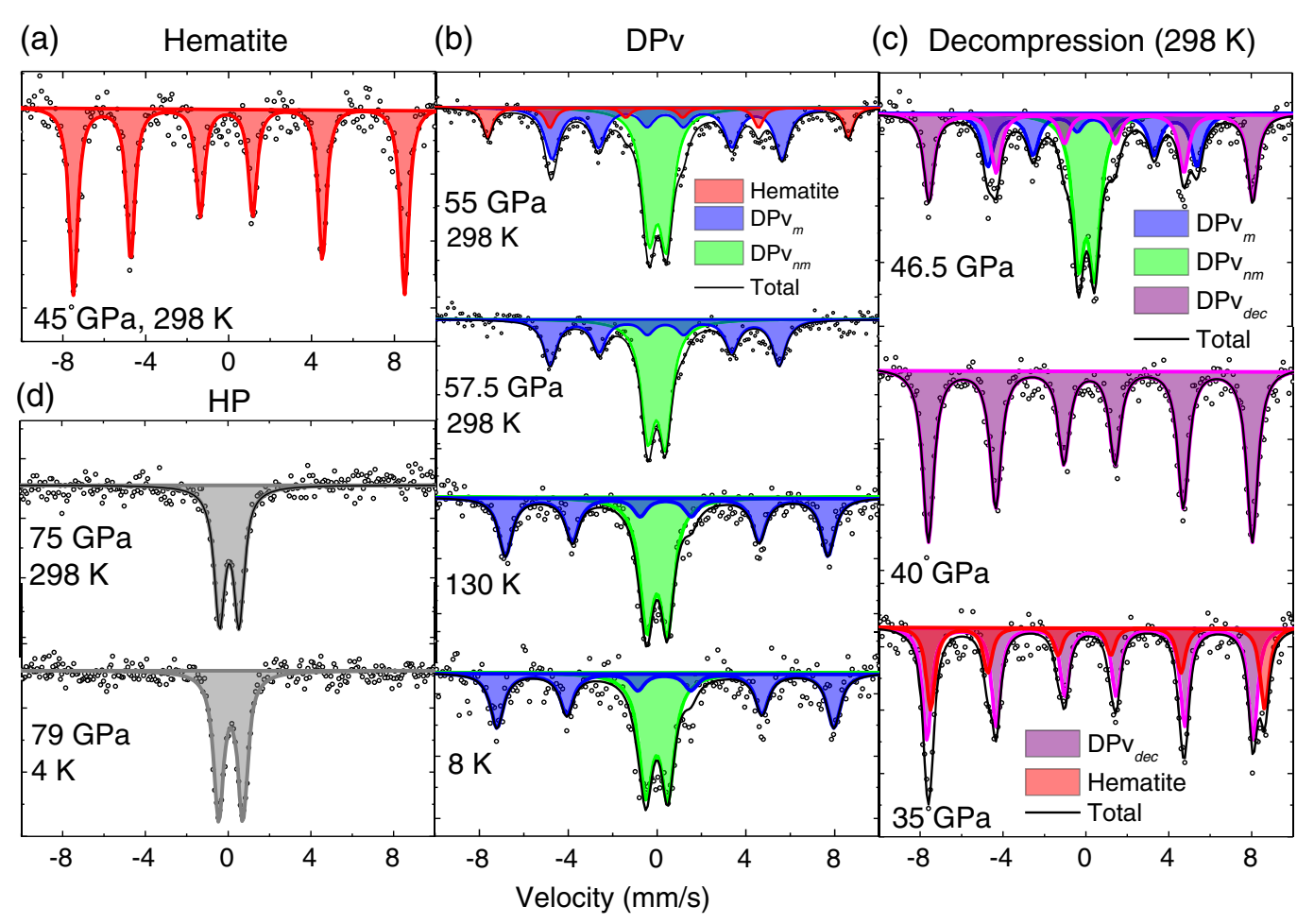

FIG. 6. ${ }^{57} \mathrm{Fe}$ Mössbauer spectra of $\mathrm{Fe}_{2} \mathrm{O}_{3}$ at various pressures and temperatures: at room temperature (a),(c) and reduced temperatures (b),(d), upon compression (a),(b),(d) and decompression (c). Red, blue, green, and magenta lines and shaded areas represent fits to the hematite, magnetic $\mathrm{DPv}\left(\mathrm{DPv}_{m}\right)$, nonmagnetic $\mathrm{DPv}\left(\mathrm{DPv}_{n m}\right)$, and $\mathrm{DPv}_{\mathrm{dec}}$ components; the black solid line is the sum of all components. The high-pressure phase (HP) component is shown in solid grey.

Upon decompression, the DPv components reappear in the MS spectra, with a hysteresis of $6 \mathrm{GPa}$ [Fig. 7(b)]. Upon further decompression, in the pressure range of $47>P>44 \mathrm{GPa}$, the two $\mathrm{DPv}$ components are replaced by a single, broader, HS component designated as $\mathrm{DPv}_{\mathrm{dec}}$ [Figs. 6(c) and S1]. However, a component with hyperfine parameters identical to those of the hematite phase appears only below $35 \mathrm{GPa}$, with a complete transition back to hematite at $25 \mathrm{GPa}$ [Figs. 6(c), and 1]. We note that decoupling of the local spin state and structural transitions in $\mathrm{Fe}_{2} \mathrm{O}_{3}$ was reported previously by Badro et al. [37].

\section{X-ray diffraction}

Powder XRD was performed at room temperature on compression to $62 \mathrm{GPa}$, followed by decompression. Our studies show that, upon compression, a first-order structural phase transition with a symmetry change takes place in the pressure range of 53-57 GPa, with a concomitant volume decrease of about 9\% (Figs. 1, 8, and S2 in [46]). Recent single-crystal diffraction $[28,36]$ shows that the phase can be described as a distorted double-perovskite-type (DPv$\mathrm{Fe}_{2} \mathrm{O}_{3}$ ) using a monoclinic unit cell with $P 2_{1} / n$ symmetry [36] (the symmetry is actually triclinic $P \overline{1}$ [28]; however, we use a monoclinic model to constrain the atomic arrangement, as done in Ref. [28]). As was mentioned above, this structure consists of a three-dimensional network of tilted corner-sharing $B^{\prime} \mathrm{O}_{6}$ and $B^{\prime \prime} \mathrm{O}_{6}$ octahedra with $A$-cations located in bicapped trigonal-prismatic voids (Fig. S3; interatomic distances are given in Table S-I [46]). The unit-cell volume as a function of pressure for hematite and $\mathrm{DPv}-\mathrm{Fe}_{2} \mathrm{O}_{3}$ is shown in Fig. 1, combining both powder and single-crystal [28] data. It is noteworthy that, upon decompression from $62 \mathrm{GPa}$, the DPv phase remains stable down to 35 GPa (Figs. 1, 8, and S2 [46]). The observed XRD spectra can be fitted well with the $P 2_{1} / n$ structure despite an appreciable change in some features around $51 \mathrm{GPa}$ (Fig. 8). Namely, at $51.3 \mathrm{GPa}$, one can clearly see a doubling of the peaks typical for a first-order isostructural phase transition with a significant volume change. An integrated pattern collected at $42.1 \mathrm{GPa}$, when the isostructural transition is completed, is shown in Fig. S2(c) [46]. In addition to the $7 \%$ volume change, the fit also shows an appreciable decrease of the monoclinic distortion of the DPv phase at pressures of 50-45 GPa (Fig. 9). It could be proposed that the latter is a consequence of leveling the volumes of $A$ and $B$ sites. Previous Raman spectroscopy measurements upon decompression [54] also suggest an isostructural transition, showing modes that remain the same but with significantly higher intensity below the transition. The structural transition back to hematite begins only below $35 \mathrm{GPa}$. According to Ref. [28], a transition to a new high-pressure polymorph is observed upon compression of the $\mathrm{DPv}-\mathrm{Fe}_{2} \mathrm{O}_{3}$ phase 


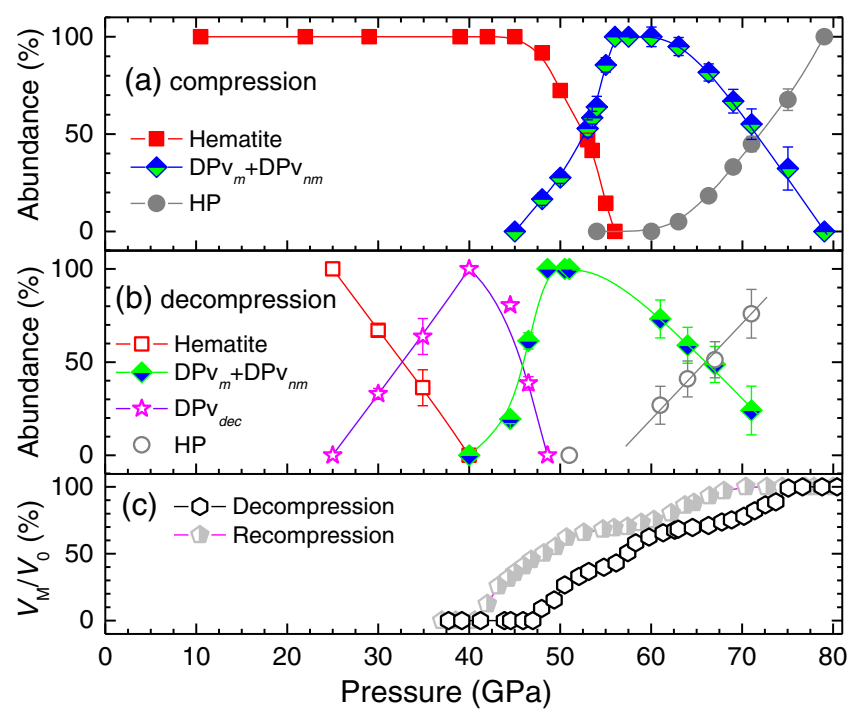

FIG. 7. Pressure evolution of the relative abundances of the Mössbauer spectral components, and the abundance of the metallic phase. The abundances of the Mössbauer spectral components are shown for compression (a) and decompression (b) as computed from the relative areas of the absorption bands. Note the onset of the new $\mathrm{DPv}_{\mathrm{dec}}$ component upon decompression below $47 \mathrm{GPa}$, with significant hysteresis in the reappearance of the $\mathrm{DPv}$ and hematite components. Upon recompression from $25 \mathrm{GPa}$ (not shown), the double-perovskite nonmagnetic $\left(\mathrm{DPv}_{n m}\right)$ and doube-perovskite magnetic $\left(\mathrm{DPv}_{m}\right)$ components appear again at $50 \mathrm{GPa}$, with a relative abundance in agreement with the compression trend. The volumetric abundance of the metallic phase is shown for decompression and recompression (from $37 \mathrm{GPa}$ ) (c), as derived from the room-temperature measurements of electrical resistance (see Supplemental Material [46]). Note the two distinct steps in the $V_{M} / V_{0}(P)$ dependence. Solid curves are guides for the eye.

above $67 \mathrm{GPa}$ (Fig. 1), with a diffraction pattern that could be indexed based on an orthorhombic Aba2 space group that has only one type of Fe cation (Fig. S3). We note, however, that the Aba2 phase observed here at room temperature is most probably metastable. Indeed, it is metastable according to our theoretical results (see above, Fig. 2). Moreover, in accordance with the previous XRD measurements [28], it disappears upon annealing to high temperatures, implying that this phase seems to appear only in the room-temperature experiments.

\section{Electrical resistance}

Our electrical resistance measurements show an abrupt 6 orders of magnitude decrease of resistance at about 40$60 \mathrm{GPa}$ upon compression (Fig. 10), in agreement with the reported insulator-metal transition at about $55 \mathrm{GPa}[23,55]$. Upon further compression, we observe a substantial change in the pressure dependence of the resistance, indicating an additional change of conductivity features at $70 \mathrm{GPa}$ [Fig. 10(a)]. Similar behavior is seen during the

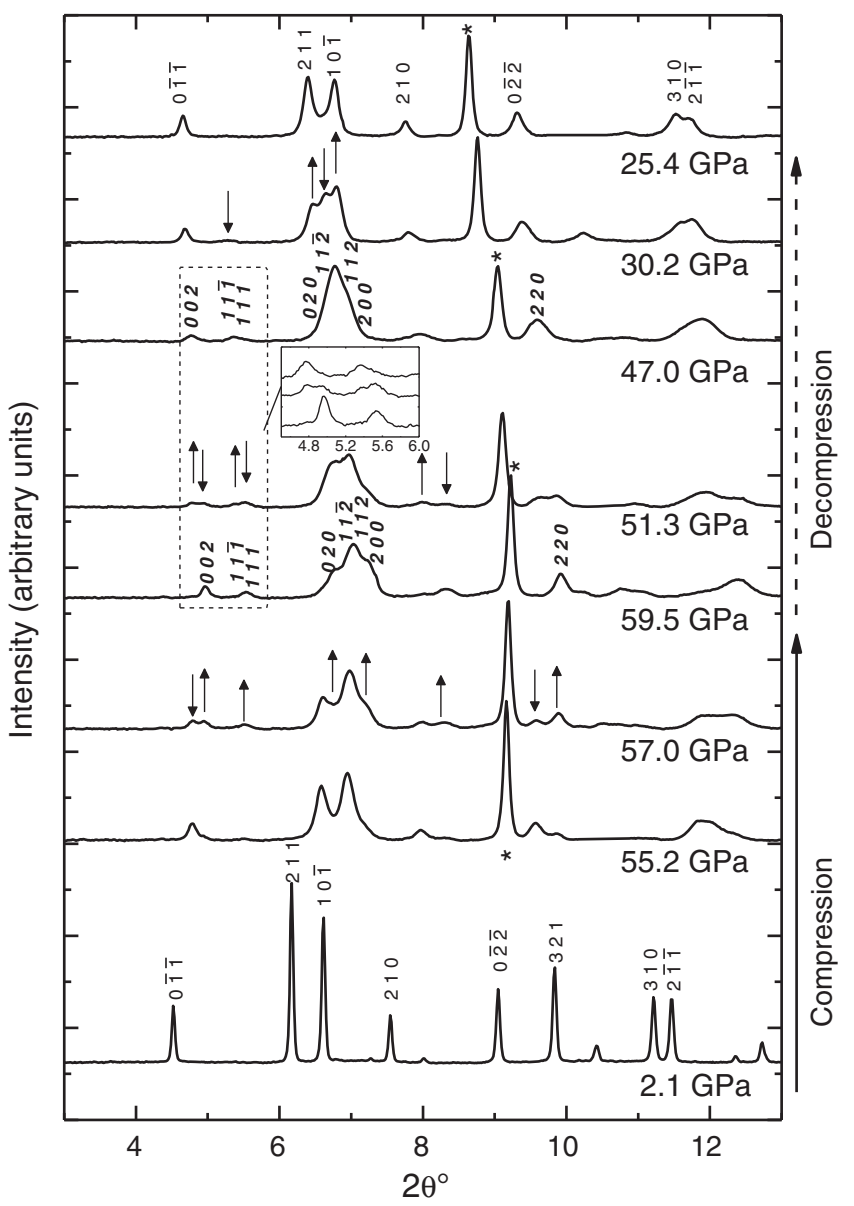

FIG. 8. X-ray powder diffraction patterns of $\mathrm{Fe}_{2} \mathrm{O}_{3}$ at $T=$ $298 \mathrm{~K}$ at various pressures. The double-perovskite (DPv) intermediate-pressure phase, which first appears at approximately $53 \mathrm{GPa}$, is clearly seen at $55.2 \mathrm{GPa}$. Up- and down-facing arrows represent an increase and decrease in the DPv and hematite phases, respectively. Upon decompression, the distinctive doubling of the diffraction peaks of the DPv phase is observed at about $51 \mathrm{GPa}$, especially (002), (111), and (11) $)$. A part of the spectrum in the $2 \theta$ range of $4.5^{\circ}-6.0^{\circ}$ is expanded in the inset to emphasize this doubling. With further decompression below $35 \mathrm{GPa}$, the peaks of the hematite $R \overline{3} c$ structure appear, and, at $25 \mathrm{GPa}$, the transition to the original hematite phase is complete. The main diffraction peak of the Ne pressure medium is marked with an asterisk. Italics correspond to the diffraction peaks of the DPv phase.

decompression cycle, with a hysteresis of about $6 \mathrm{GPa}$. It is noteworthy that, upon pressure release, the resistance rises only by 3 orders of magnitude at about $50-40 \mathrm{GPa}$, saturating below $40 \mathrm{GPa}$. Furthermore, to avoid a structural transition back to the corundum structure, we terminated decompression at $37 \mathrm{GPa}$ and performed recompression measurements up to $83 \mathrm{GPa}$. The pressure-temperature dependence of electrical resistance upon recompression shows an abrupt drop at 45-60 GPa, with the onset of metallization at around 53 GPa [Fig. 10(b)]. Similar to the hematite phase [55], the temperature dependence of the resistance of the insulating 


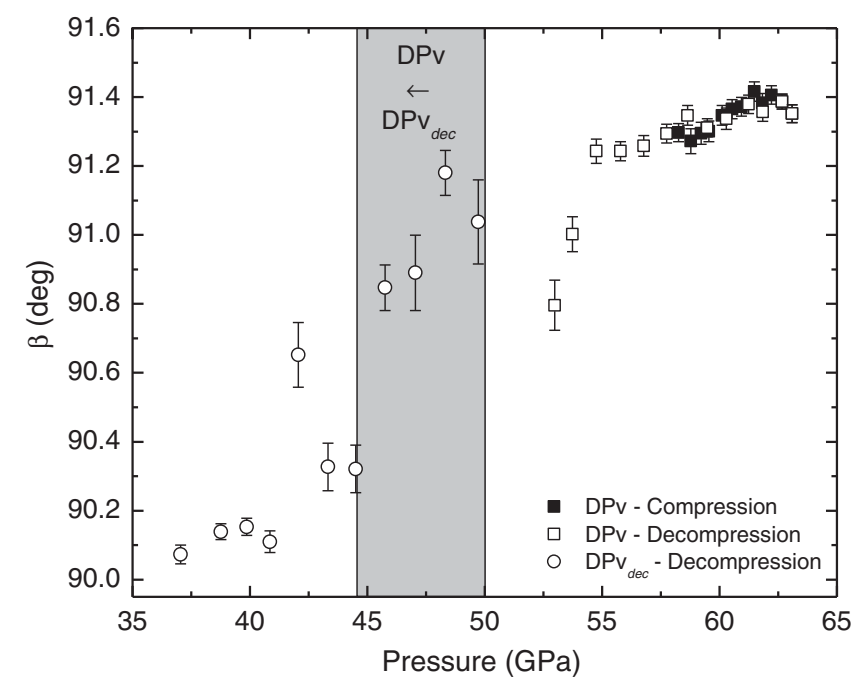

FIG. 9. Monoclinic distortion of the double-perovskite phase of $\mathrm{Fe}_{2} \mathrm{O}_{3}$. Pressure evolution of the $\beta$ angle, characteristic of the monoclinic distortion of the unit cell with $P 2_{1} / n$ symmetry (DPv structure), upon compression and decompression (solid and open symbols, respectively). Note the significant decrease of the monoclinic distortion coinciding with the reverse electronic transition to the strongly correlated state upon decompression; the $\beta$ angle changes from $91.4^{\circ}$ to $90.1^{\circ}$.

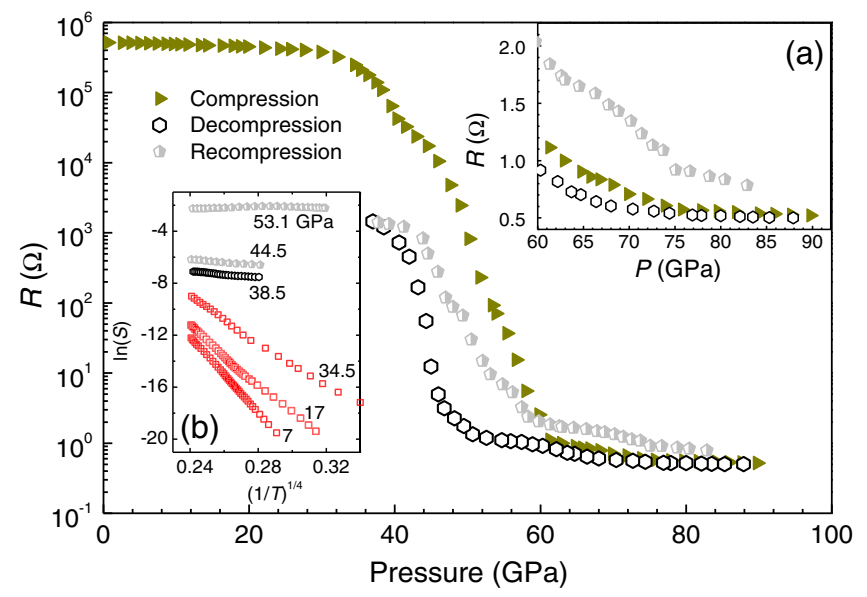

FIG. 10. Pressure dependence of electrical resistance at $298 \mathrm{~K}$. The solid triangles, open hexagons, and half-filled pentagons show data recorded upon compression, decompression, and successive recompression, respectively. Inset (a): our results for pressures of 60-90 GPa; inset (b): temperature dependence of electrical conductance $S$ of $\mathrm{Fe}_{2} \mathrm{O}_{3}$, with measurements on the $\mathrm{DPv}$ phase performed during decompression to $37 \mathrm{GPa}$, followed by recompression. Measurements on the hematite phase were collected during a separate decompression cycle to ambient pressure (symbols "square") [55]. The change in sign of the slope $\operatorname{d} \ln (S) / d T$ documented at approximately $53 \mathrm{GPa}$ is the signature of metallic conductivity.
$\mathrm{DPv}$ phase is associated with a variable-range hopping mechanism below $50 \mathrm{GPa}$ : The electrical conductivity varies as $\sigma=C \exp \left(T_{0} / T\right)^{1 / 4}$, though we notice a significantly reduced Mott temperature value $T_{0}$ [Figs. 10(b) and S4(b)]. Meanwhile, within the metallic region above $50 \mathrm{GPa}$, the resistance exhibits a clear deviation from Fermi-liquid-like $T^{2}$ behavior, showing a minimum at temperature $T=$ $110-150 \mathrm{~K}$ for the DPv phase and at about $75 \mathrm{~K}$ for the HP phase [Fig. S4(a); for details, see the Supplemental Material [46]). This behavior is in good agreement with the computation results, namely, that the DFT + DMFT calculations predict a substantial enhancement of the effective mass of $3 d$ electrons and show a sharp Kondo-like peak at the Fermi level. Furthermore, this is consistent with the absence of magnetic correlations of the $\mathrm{Fe} B$ sites in the MS spectra down to the lowest measured temperatures (Fig. 6). That is, the $\mathrm{Fe} B$ electrons are delocalized, the corresponding longtime magnetic susceptibilities are well screened, and the (instantaneous) amplitude of the fluctuating moments is small. However, it is noteworthy that an observation of minima in $R(T)$ does not necessarily imply the Kondo effect: The electron-electron interaction and localization effects may result in a similar behavior [56,57].

\section{DISCUSSION}

Summarizing our theoretical and experimental results, we find evidence that the metallization transition in $\mathrm{Fe}_{2} \mathrm{O}_{3}$ occurs in stages with pressure, first for half the Fe cations in the DPv phase - those in the octahedral $B^{\prime}$ and $B^{\prime \prime}$ sites with the collapsed magnetic moment $\left(\mathrm{DPv}_{n m}\right)$, while the prismatic Fe $A$ sites remain insulating and high spin, and then for all the $\mathrm{Fe}$ in the high-pressure (metastable) $\mathrm{Aba} 2$ or $\mathrm{PPv}$ structure. Labeling iron ions contributing electrons to the conduction band as $\mathrm{Fe}^{M}$, we summarize the transitions as $\left({ }^{\mathrm{VI}} \mathrm{Fe}^{3+\mathrm{HS}}\right)_{2} \mathrm{O}_{3}[R \overline{3} c] \rightarrow\left({ }^{\mathrm{VIII}} \mathrm{Fe}^{3+\mathrm{HS} \mathrm{VI}} \mathrm{Fe}^{\mathrm{M}}\right) \mathrm{O}_{3}\left[P 2_{1} / n\right] \rightarrow$ $\left({ }^{\mathrm{VI}} \mathrm{Fe}^{\mathrm{M}}\right)_{2} \mathrm{O}_{3}[\mathrm{Aba2} / \mathrm{PPv}]$ with increasing pressure, where subscript Roman numerals indicate nearest-neighbor $(\mathrm{Fe}-\mathrm{O})$ coordination and crystal structures are given in brackets (HS designates high spin for the ferric ion). The average $\mathrm{Fe}-\mathrm{O}$ bond length collapses upon metallization, from $1.91 \AA$ in hematite at $51 \mathrm{GPa}$ to $1.82 \AA$ in the highpressure $A b a 2$ phase at $74 \mathrm{GPa}$; in between, it is the coexistence of small octahedral sites along with large eight-coordinated sites in the DPv structure that allows for the site-selective insulator-metal transition (Table S-I [46]). We have demonstrated that theoretical calculations are in accordance with our Mössbauer, XRD, and electrical resistance measurements. In particular, we have shown that the electronic and structural transitions to the $R \overline{3} c$ phase do not coincide upon decompression, but are separated by a pressure interval of about $20 \mathrm{GPa}$. Overall, our theoretical results for the electronic structure, equilibrium lattice constant, and phase stability of paramagnetic $\mathrm{Fe}_{2} \mathrm{O}_{3}$ agree remarkably well with available experiments. 
The three-dimensional network of the octahedral Fe $B$ sites forms a high-mobility path that causes resistance to drop by many orders of magnitude, compared to the hematite phase, even though only half the sites contribute. For instance, we expect a filamentary conductivity in the three-dimensional network of Fe octahedral sites, analogous to the conductance path for a percolation threshold. At higher pressures, the trigonal-prismatic sites also contribute to the electron mobility, but the effect is only a quantitative further decrease in resistance, by a factor of 2 . The volume change upon metallization is identical to that observed in $\mathrm{CaFe}_{2} \mathrm{O}_{4}$ [58], suggesting a similar mechanism of electronic transition for these sites: namely, a spin transition resulting in complete closure of the Mott-Hubbard gap $[7,11,12]$ in accordance with our theoretical calculations.

We note that metallization does not occur in the hematite ( $R \overline{3} c$ phase upon compression). In the region where the MS data find both the hematite and the DPv phases, the remaining hematite phase is still in a high-spin state, and no appreciable change in the Mössbauer parameter values was observed (even when half of the Fe in the DPv phase are already nonmagnetic, metallic). In addition, the $V(P)$ data in Fig. 1 show that there is no appreciable change in unit-cell volume of hematite during compression (no deviation from the hematite EOS), even in the region of coexistence. This is in full accordance with our theoretical calculations, which show that, for the hematite phase, the IM transition associated with a HS-LS state transformation would take place at a pressure of $72 \mathrm{GPa}\left(V \sim 0.74 V_{0}\right)$ (Fig. S5 [46]).

Upon decompression, we observe (theoretically and experimentally) a sharp reversal in electronic properties at about $45 \mathrm{GPa}$, with a metal-to-insulator transition and retrieval of a magnetic state [Figs. 6(c) and 10], which can be described by $\left({ }^{\mathrm{VIII}} \mathrm{Fe}^{3+\mathrm{HSVI}} \mathrm{Fe}^{\mathrm{M}}\right) \mathrm{O}_{3}\left[P 2_{1} / n\right] \rightarrow$ $\left({ }^{\mathrm{VIII}} \mathrm{Fe}^{3+\mathrm{HSVI}} \mathrm{Fe}^{3+\mathrm{HS}}\right) \mathrm{O}_{3}\left[P 2_{1} / n\right]$. The $\mathrm{DPv}$ structure remains unchanged and only the volume increases by about $7 \%$, however, documenting the decoupling of electronic and structural phase transitions. This implies that, upon decompression, $\mathrm{Fe}_{2} \mathrm{O}_{3}$ undergoes an isostructural site-selective Mott transition, not complicated by any coinciding structural transformations. We conclude that we are documenting intrinsic electronic properties of the DPv phase of $\mathrm{Fe}_{2} \mathrm{O}_{3}$, as indicated by the small hysteresis in electrical resistance upon decompression and recompression near the onset of the insulator-metal transition (contrast the recompression results for hematite in previous studies $[23,55])$. Interestingly, the isostructural transition results in a relatively small change of the bulk modulus to $258 \mathrm{GPa}$, which is comparable to that of the LS state of the $R \overline{3} c$ phase. The structural transition from DPv back to the original corundum-type structure takes place only below 35 GPa (Figs. 1 and 8).

The site-selective Mott transition that we document differs from the "orbital-selective" Mott phase that has been proposed for multiorbital transition-metal oxides $[9,10]$. In the orbital-selective phase, because of the inclusion of orbital degrees of freedom, a partial localization can take place, in which some orbitals are conducting, while others are localized. As a result, localized spins and itinerant electrons can coexist. In contrast to materials with orbitalselective state(s), the reason for the appearance of an intermediate electronic state in $\mathrm{Fe}_{2} \mathrm{O}_{3}$, with a coexistence of localized (HS) and itinerant (nonmagnetic) Fe $3 d$ electrons, is the formation of two, distinct (sixfold and eightfold) coordination environments.

We expect that this combination of localized and itinerant $3 d$ electrons can give rise to a complex electronic state of $\mathrm{DPv} \mathrm{Fe}_{2} \mathrm{O}_{3}$ at low temperatures, e.g., resulting in heavy-fermion-like behavior associated with the Kondo effect: This still needs to be clarified. Indeed, our experimental transport data exhibit a Kondo-like abnormal behavior of resistance at approximately 110-150 K, while our calculations predict a substantial enhancement of the effective mass of $3 d$ electrons and show a sharp Kondo-like peak at the Fermi level. This behavior is consistent with the observed absence of magnetic correlations of the Fe $B$ sites in the MS spectra down to the lowest measured temperatures (Fig. 6). The Fe $B$ electrons are delocalized, the corresponding long-time magnetic susceptibilities are well screened, and the (instantaneous) amplitude of the fluctuating moments is small. Because of that, no magnetic response can be detected by a relatively slow probe such as Mössbauer spectroscopy. We note, however, that a Kondolike minimum in resistance can be alternatively explained by the presence of weak localization due to disorder at the onset of a Mott insulator-to-metal phase transition under pressure [56,57].

The appearance of the $P 2_{1} / n \mathrm{DPv}$ phase can be understood as a result of the interplay between cohesive (lattice) energy and local magnetic moments. While the former favors the denser high-pressure phase (e.g., Aba2), the local magnetic moments enter into the total energy as $-I\left\langle m_{z}^{2}\right\rangle / 4$ (here, $I$ is a Stoner exchange interaction, and $\left\langle m_{z}^{2}\right\rangle$ is the square of the local magnetic moment), and, therefore, favor the corundum-structured $(R \overline{3} c)$ phase, i.e., the phase with high local magnetic moments. As a result, the intermediate DPv phase, with site-selective electronic and magnetic properties, is stabilized at intermediate pressures of about 50-60 GPa. This is presumably an electronic phase transition that results in the appearance of (at least) two electronically/magnetically different sublattices of Fecations; i.e., it leads to a structural transformation, due to electron-lattice coupling. Similar behavior only associated with the site-selective HS-LS transition is found to occur in a two-orbital Hubbard model with crystal-field splitting [59]. Meanwhile, during decompression we observe the "pure" electronic transition back to a conventional Mott insulating state within the DPv phase, with decoupling of the electronic and lattice (crystal-structure) degrees of 
freedom. This behavior is similar to what happens in Fe-bearing bridgmanite $\left(\mathrm{MgSiO}_{3}\right.$-peroskite), where only half of the $\mathrm{Fe}^{3+}$ (those in the $B$ site) undergo a HS-to-LS transition under pressure, while $\mathrm{Fe}^{3+}$ in the $A$ site remains in the HS state up to at least $100 \mathrm{GPa}[18,19]$. We note, however, the important difference that in $\mathrm{Fe}_{2} \mathrm{O}_{3}$ upon decompression the LS-HS transition is accompanied by a transformation to the conventional Mott insulating phase.

Upon compression of $\mathrm{Fe}_{2} \mathrm{O}_{3}$ above $62 \mathrm{GPa}$, we observe a further increase in the abundance of a nonmagnetic component of the $\mathrm{Fe}^{3+}$ cations, which is presumably caused by the onset of the Fe $A$ sites of the DPv structure transforming into a metallic nonmagnetic state. This results in a structural transition from the DPv to a HP phase, corresponding to completion of the electronic transition. According to Ref. [28], the HP phase at room temperature has the orthorhombic Aba2 structure characterized by a single cation position. However, Ref. [28] and our DFT + DMFT results indicate that this phase is metastable, and, upon annealing, it is found to collapse into the $\mathrm{PPv}$ structure, the most stable polymorph above approximately $75 \mathrm{GPa}$ (see Figs. 2 and 1). Correspondingly, at higher temperatures, one may expect also a direct $\mathrm{DPv} \rightarrow \mathrm{PPv}$ phase transition. Importantly, in both cases ( $\mathrm{DPv} \rightarrow A b a 2$ or $\mathrm{PPv}$ structure), the site-selective Mott insulator $\mathrm{Fe}_{2} \mathrm{O}_{3}$ transforms into a conventional metallic state: In the case of the $\mathrm{PPv}$ phase, all crystallographic Fe sites are metallic. Thus, starting from a structure with a single crystallographic site for $\mathrm{Fe}^{3+}$ cations, $\mathrm{Fe}_{2} \mathrm{O}_{3}$ transforms into an intermediate structure containing multiple $\mathrm{Fe}$ sites, half of which are metallic, before the tendency of $\mathrm{Fe}^{3+}$ to metallize upon compression results in a second structural transition, to the conventional metallic phase (single- or multisited).

Summing up our theoretical and experimental results for $\mathrm{Fe}_{2} \mathrm{O}_{3}$, our study demonstrates the complexity of electronic and structural transformations that can arise in strongly correlated transition-metal compounds undergoing a Mott insulator-to-metal transition. Interestingly, the electronic and structural behavior of $\mathrm{Fe}_{2} \mathrm{O}_{3}$ under pressure is found to be complicated by the appearance of metastable phases, e.g., the $\mathrm{Rh}_{2} \mathrm{O}_{3}$-II-type and $\mathrm{Aba2}$ phases (at high and low temperatures, respectively), and this topic deserves further consideration.

Our results suggest that the concept of a site-selective Mott transition may be broadly applicable to correlatedelectron materials: in particular, in those with a corundum crystal structure, as in the case of $\mathrm{Fe}_{2} \mathrm{O}_{3}$. For example, in $\mathrm{Mn}_{2} \mathrm{O}_{3}$, corundum-type $\varepsilon-\mathrm{Mn}_{2} \mathrm{O}_{3}$ (and below $T=1200 \mathrm{~K}$ cubic $\alpha-\mathrm{Mn}_{2} \mathrm{O}_{3}$ ) transforms upon compression to a distorted perovskite structure [60], and this structural transition coincides with an insulator-metal transition [61]. Similar electronic transformations could be expected in other sesquioxides, e.g., $\mathrm{Cr}_{2} \mathrm{O}_{3}$ [62] or $\mathrm{Ti}_{2} \mathrm{O}_{3}$ [63], and in materials with a complex crystal structure (or that acquire a complex structure under pressure) containing transition- metal cations in different coordination polyhedra: for example, in magnetite [14] or Fe-bearing bridgmanite $[18,19]$. Thus, such effect(s) can occur in crystalline oxides comprising Earth and planetary mantles. Indeed, the major components of Earth's lower mantle-bridgmanite and ferropericlase-contain ferric or ferrous iron (or both) and, therefore, changes of the electronic state in such materials likely affect the properties of our planet's deep interior $[64,65]$.

\section{ACKNOWLEDGMENTS}

We would like to thank Professor D. Vollhardt and Dr. W. $\mathrm{Xu}$ for valuable discussions, Dr. R. Rüffer for experimental assistance with the facilities of the ID18 beam line at ESRF, and Dr. A. Kurnosov for loading of DACs with Ne gas. This research was supported in part by Israeli Science Foundation Grant No. 1189/14, and also in part by the U.S. Department of Energy. Financial support from the Swedish Research Council (VR) through Grant No. 2015-04391, the Swedish Government Strategic Research Area in Materials Science on Functional Materials at Linköping University (Faculty Grant SFOMatLiU No. 2009 00971), the Swedish e-Science Research Centre (SeRC), and the Deutsche Forschungsgemeinschaft through Transregio TRR 80 is gratefully acknowledged. Theoretical analysis of structural properties was supported by the Russian Science Foundation (Project No. 18-12-00492). Simulations of the electronic structure were supported by the Ministry of Education and Science of the Russian Federation in the framework of Increase Competitiveness Program of NUST "MISIS" (No. K3-2016-027), implemented by a governmental decree, No. 211.

E. G. and I. L. contributed equally to this work.

[1] N. F. Mott, Metal-Insulator Transitions (Taylor \& Francis, London, 1990).

[2] M. Imada, A. Fujimori, and Y. Tokura, Metal-Insulator Transitions, Rev. Mod. Phys. 70, 1039 (1998).

[3] Q. Si and E. Abrahams, Strong Correlations and Magnetic Frustration in the High Tc Iron Pnictides, Phys. Rev. Lett. 101, 076401 (2008).

[4] L. de'Medici, G. Gianluca, and M. Capone, Selective Mott Physics as a Key to Iron Superconductors, Phys. Rev. Lett. 112, 177001 (2014).

[5] G. Kh. Rozenberg, W. Xu, and M. P. Pasternak, The Mott Insulators at Extreme Conditions; Structural Consequences of Pressure-Induced Electronic Transitions, Z. Kristallogr. 229, 210 (2014).

[6] I. Leonov, V. I. Anisimov, and D. Vollhardt, Metal-Insulator Transition and Lattice Instability of Paramagnetic $\mathrm{V}_{2} \mathrm{O}_{3}$, Phys. Rev. B 91, 195115 (2015).

[7] J. Kuneš, A. V. Lukoyanov, V. I. Anisimov, R. T. Scalettar, and W. E. Pickett, Collapse of Magnetic Moment Drives the Mott Transition in MnO, Nat. Mater. 7, 198 (2008). 
[8] I. Leonov, L. Pourovskii, A. Georges, and I. A. Abrikosov, Magnetic Collapse and the Behavior of Transition Metal Oxides at High Pressure, Phys. Rev. B 94, 155135 (2016).

[9] V. I. Anisimov, I. A. Nekrasov, D. E. Kondakov, T. M. Rice, and M. Sigrist, Orbital-Selective Mott-Insulator Transition in $\mathrm{Ca}_{2-x} \mathrm{Sr}_{x} \mathrm{RuO}_{4}$, Eur. Phys. J. B 25, 191 (2002).

[10] L. de'Medici, A. Georges, and S. Biermann, OrbitalSelective Mott Transition in Multiband Systems: Slave-Spin Representation, and Dynamical Mean-Field Theory, Phys. Rev. B 72, 205124 (2005).

[11] I. S. Lyubutin, S. G. Ovchinnikov, A. G. Gavriliuk, and V. V. Struzhkin, Spin-Crossover-Induced Mott Transition and the Other Scenarios of Metallization in $3 d^{n}$ Metal Compounds, Phys. Rev. B 79, 085125 (2009).

[12] E. Greenberg, G. Kh. Rozenberg, W. Xu, M. P. Pasternak, C. McCammon, K. Glazyrin, and L.S. Dubrovinsky, Mott Transition in $\mathrm{CaFe}_{2} \mathrm{O}_{4}$ at around 50 GPa, Phys. Rev. B 88, 214109 (2013).

[13] A. G. Gavriliuk, V. V. Struzhkin, I. S. Lyubutin, S. G. Ovchinnikov, M. Y. Hu, and P. Chow, Another Mechanism for the Insulator-Metal Transition Observed in Mott Insulators, Phys. Rev. B 77, 155112 (2008).

[14] E. Greenberg, W. Xu, M. Nikolaevsky, E. Bykova, G. Garbarino, K. Glazyrin, D. G. Merkel, L. Dubrovinsky, M. P. Pasternak, and G. Kh. Rozenberg, High-Pressure Magnetic, Electronic and Structural Properties of $\mathrm{MFe}_{2} \mathrm{O}_{4}$ ( $M=\mathrm{Mg}, \mathrm{Zn}, \mathrm{Fe}$ ) Ferric Spinels, Phys. Rev. B 95, 195150 (2017).

[15] W. M. Xu, G. R. Hearne, S. Layek, D. Levy, J.-P. Itié, M. P. Pasternak, G. Kh. Rozenberg, and E. Greenberg, $\mathrm{FeCr}_{2} \mathrm{O}_{4}$ Spinel to near Megabar Pressures: Orbital Moment Collapse and Site-Inversion Facilitated Spin Crossover, Phys. Rev. B 95, 045110 (2017).

[16] W. M. Xu, G. R. Hearne, S. Layek, D. Levy, J.-P. Itié, M. P. Pasternak, G. Kh. Rozenberg, and E. Greenberg, SiteSpecific Spin Crossover in $\mathrm{Fe}_{2} \mathrm{TiO}_{4}$ Post-Spinel under High Pressure up to Nearly a Megabar, Phys. Rev. B 96, 045108 (2017).

[17] W. M. Xu, G. R. Hearne, S. Layek, D. Levy, J.-P. Itié, M. P. Pasternak, G. Kh. Rozenberg, and E. Greenberg, Interplay between Structural and Magnetic-Electronic Responses of $\mathrm{FeAl}_{2} \mathrm{O}_{4}$ to a Megabar: Site Inversion and Spin Crossover, Phys. Rev. B 97, 085120 (2018).

[18] K. Catalli, S.-H. Shim, V. B. Prakapenka, J. Zhao, W. Sturhahn, P. Chow, Y. Xiao, H. Liu, H. Cynn, and W. J. Evans, Spin State of Ferric Iron in $\mathrm{MgSiO}_{3}$ Perovskite and Its Effect on Elastic Properties, Earth Planet. Sci. Lett. 289, 68 (2010).

[19] H. Hsu, P. Blaha, M. Cococcioni, and R. M. Wentzcovitch, Spin-State Crossover and Hyperfine Interactions of Ferric Iron in $\mathrm{MgSiO}_{3}$ Perovskite, Phys. Rev. Lett. 106, 118501 (2011).

[20] H. Park, A. J. Millis, and C. A. Marianetti, Site-Selective Mott Transition in Rare-Earth-Element Nickelates, Phys. Rev. Lett. 109, 156402 (2012).

[21] A. Subedi, O. E. Peil, and A. Georges, Low-Energy Description of the Metal-Insulator Transition in the Rare-Earth Nickelates, Phys. Rev. B 91, 075128 (2015).
[22] T. Suzuki et al., Solid State Physics under Pressure, edited by S. Minomura (Terra Scientific Publishing Company, Tokyo, 1985), pp. 149-154.

[23] M. P. Pasternak, G. Kh. Rozenberg, G. Yu. Machavariani, O. Naaman, R. D. Taylor, and R. Jeanloz, Breakdown of the Mott-Hubbard State in $\mathrm{Fe}_{2} \mathrm{O}_{3}$ : A First-Order InsulatorMetal Transition with Collapse of Magnetism at $50 \mathrm{GPa}$, Phys. Rev. Lett. 82, 4663 (1999).

[24] Y. Syono, A. Ito, S. Morimoto, T. Suzuki, T. Yagi, and S.-i. Akimoto, Mössbauer Study on the High Pressure Phase of $\mathrm{Fe}_{2} \mathrm{O}_{3}$, Solid State Commun. 50, 97 (1984).

[25] S. Nasu, K. Kurimoto, S. Nagatomo, S. Endo, and F. E. Fujita, ${ }^{57} \mathrm{Fe}$ Mössbauer Study under High Pressure; $\varepsilon$-Fe and $\mathrm{Fe}_{2} \mathrm{O}_{3}$, Hyperfine Interact. 29, 1583 (1986).

[26] J. Badro, V. V. Struzhkin, J. Shu, R. J. Hemley, H.-k. Mao, C.-c. Kao, J.-P. Rueff, and G. Shen, Magnetism in FeO at Megabar Pressures from X-Ray Emission Spectroscopy, Phys. Rev. Lett. 83, 4101 (1999).

[27] S. Wang et al., High Pressure Evolution of $\mathrm{Fe}_{2} \mathrm{O}_{3}$ Electronic Structure Revealed by X-ray Absorption, Phys. Rev. B 82, 144428 (2010).

[28] E. Bykova et al., Structural Complexity of Simple $\mathrm{Fe}_{2} \mathrm{O}_{3}$ at High Pressures and Temperatures, Nat. Commun. 7, 10661 (2016).

[29] A. Fujimori, M. Saeki, N. Kimizuka, M. Taniguchi, and S. Suga, Photoemission Satellites and Electronic Structure of $\mathrm{Fe}_{2} \mathrm{O}_{3}$, Phys. Rev. B 34, 7318 (1986).

[30] R. G. McQueen and S. P. Marsh, in Handbook of Physical Constants, edited by S. P. Clark (Geological Society of America, Inc., New York, 1966), revised edition, p. 153.

[31] J. S. Olsen, C. S. G. Cousins, L. Gerward, H. Jhans, and B. J. Sheldon, A Study of the Crystal Structure of $\mathrm{Fe}_{2} \mathrm{O}_{3}$ in the Pressure Range up to $65 \mathrm{GPa}$ Using Synchrotron Radiation, Phys. Scr. 43, 327 (1991).

[32] G. Kh. Rozenberg, L. S. Dubrovinsky, M. P. Pasternak, O. Naaman, T. Le Bihan, and R. Ahuja, High-Pressure Structural Studies of Hematite $\mathrm{Fe}_{2} \mathrm{O}_{3}$, Phys. Rev. B 65 , 064112 (2002).

[33] T. Yagi and S. Akimoto, in High Pressure Research in Geophysics, edited by S. Akimoto and M. H. Manghnani (Center Academic Publishers, Tokyo, 1982), p. 81.

[34] E. Knittle and R. Jeanloz, High-Pressure Electrical Resistivity Measurements of $\mathrm{Fe}_{2} \mathrm{O}_{3}$ : Comparison of StaticCompression and Shock-Wave Experiments to $61 \mathrm{GPa}$, Solid State Commun. 58, 129 (1986).

[35] A. F. Reid and A. E. Ringwood, High-Pressure Scandium Oxide and Its Place in the Molar Volume Relationships of Dense Structures of $\mathrm{M}_{2} \mathrm{X}_{3}$ and $\mathrm{ABX}_{3}$ Type, J. Geophys. Res. 74, 3238 (1969).

[36] E. Bykova, M. Bykov, V. Prakapenka, Z. Konôpková, H.-P. Liermann, N. Dubrovinskaia, and L. Dubrovinsky, Novel High Pressure Monoclinic $\mathrm{Fe}_{2} \mathrm{O}_{3}$ Polymorph Revealed by Single-Crystal Synchrotron X-ray Diffraction Studies, High Press. Res. 33, 534 (2013).

[37] J. Badro, G. Fiquet, V. V. Struzhkin, M. Somayazulu, H.-k. Mao, G. Shen, and T. Le Bihan, Nature of the High-Pressure Transition in $\mathrm{Fe}_{2} \mathrm{O}_{3}$ Hematite, Phys. Rev. Lett. 89, 205504 (2002). 
[38] A. Sanson et al., Local Structure and Spin Transition in $\mathrm{Fe}_{2} \mathrm{O}_{3}$ Hematite at High Pressure, Phys. Rev. B 94, 14112 (2016).

[39] A. Georges, G. Kotliar, W. Krauth, and M. J. Rozenberg, Dynamical Mean-Field Theory of Strongly Correlated Fermion Systems and the Limit of Infinite Dimensions, Rev. Mod. Phys. 68, 13 (1996).

[40] J. Kuneš, D. M. Korotin, M. A. Korotin, V. I. Anisimov, and P. Werner, Pressure-Driven Metal-Insulator Transition in Hematite from Dynamical Mean-Field Theory, Phys. Rev. Lett. 102, 146402 (2009).

[41] L. V. Pourovskii, B. Amadon, S. Biermann, and A. Georges, Self-Consistency over the Charge Density in Dynamical Mean-field Theory: A Linear Muffin-Tin Implementation and Some Physical Implications, Phys. Rev. B 76, 235101 (2007).

[42] I. Leonov, Dm. Korotin, N. Binggeli, V. I. Anisimov, and D. Vollhardt, Computation of Correlation-Induced Atomic Displacements and Structural Transformations in Paramagnetic $\mathrm{KCuF}_{3}$ and $\mathrm{LaMnO}_{3}$, Phys. Rev. B 81, 075109 (2010).

[43] G. Trimarchi, I. Leonov, N. Binggeli, Dm. Korotin, and V. I. Anisimov, LDA + DMFT Implemented with the Pseudopotential Plane-Wave Approach, J. Phys. Condens. Matter 20, 135227 (2008).

[44] P. Giannozzi, S. Baroni, N. Bonini, M. Calandra, R. Car et al., QUANTUM ESPRESSO: A Modular and OpenSource Software Project for Quantum Simulations of Materials, J. Phys. Condens. Matter 21, 395502 (2009).

[45] E. Gull, A. J. Millis, A. I. Lichtenstein, A. N. Rubtsov, M. Troyer, and P. Werner, Continuous-Time Monte Carlo Methods for Quantum Impurity Models, Rev. Mod. Phys. 83, 349 (2011).

[46] See Supplemental Material at http://link.aps.org/ supplemental/10.1103/PhysRevX.8.031059 for a detailed discussion of the electronic structure, magnetic state, and lattice properties of $\mathrm{Fe}_{2} \mathrm{O}_{3}$ under pressure, which includes Refs. [47-53].

[47] V. I. Anisimov et al., Full Orbital Calculation Scheme for Materials with Strongly Correlated Electrons, Phys. Rev. B 71, 125119 (2005).

[48] C. Prescher, C. McCammon, and L. Dubrovinsky, Moss A: A Program for Analyzing Energy-Domain Mössbauer Spectra from Conventional and Synchrotron Sources, J. Appl. Crystallogr. 45, 329 (2012).

[49] A. P. Hammersley, ESRF Internal Report, ESRF98HA01T, FIT2D V9.129 Reference Manual V3.1 (ESRF, Grenoble, France, 1998).

[50] A. C. Larson and R. B. Von Dreele, General Structure Analysis System (GSAS), Los Alamos National Laboratory (LAUR, Los Alamos, 1994), p. 86.

[51] H. T. Brian, EXPGUI, A Graphical User Interface for GSAS, J. Appl. Crystallogr. 34, 210 (2001).
[52] P. L. Rossiter, The Electrical Resistivity of Metals and Alloys, edited by R. W. Cahn, E. A. Davis, and I. M. Ward (Cambridge University Press, Cambridge, 1991).

[53] O. L. Anderson, Equations of State of Solids for Geophysics and Ceramic Science (Oxford, New York, 1995), p. 405.

[54] S. H. Shim and T. S. Duffy, Raman Spectroscopy of $\mathrm{Fe}_{2} \mathrm{O}_{3}$ to 62 GPa, Am. Mineral. 87, 318 (2002).

[55] G. Yu. Machavariani, M. P. Pasternak, and G. Kh. Rozenberg, High Pressure Metallization of Hematite, in Science and Technology of High Pressure V2, Proceedings of the International Conference on High Pressure Science and Technology (AIRAPT-17), AIRAPT, Honolulu, Hawii, 25-30 July 1999 (Universities Press, Hyderabad, India, 2000), pp. 562.

[56] T. Zingg, T. Richomond, and H. J. Güntherodt, Negative Temperature Coefficient of Conductivity at Low Temperatures in Metallic Glasses: Experimental Evidence for Electron-Electron Interaction, Europhys. Lett. 9, 471 (1989).

[57] A. Das and A. K. Majumdar, High-Temperature Resistivity Minima in Co-rich Amorphous Ferromagnets, Phys. Rev. B 43, 6042 (1991).

[58] M. Merlini, M. Hanfland, M. Gemmi, S. Huotari, L. Simonelli, and P. Strobel, $\mathrm{Fe}^{3+}$ Spin Transition in $\mathrm{CaFe}_{2} \mathrm{O}_{4}$ at High Pressure, Am. Mineral. 95, 200 (2010).

[59] J. Kuneś and V. Kŕápek, Disproportionation and Metallization at Low-Spin to High-Spin Transition in Multiorbital Mott Systems, Phys. Rev. Lett. 106, 256401 (2011).

[60] S. V. Ovsyannikov, A. M. Abakumov, A. A. Tsirlin, W. Schnelle, R. Egoavil, J. Verbeeck, G. Van Tendeloo, K. V. Glazyrin, M. Hanfland, and L. Dubrovinsky, PerovskiteLike $\mathrm{Mn}_{2} \mathrm{O}_{3}$ : A Path to New Manganites, Angew. Chem., Int. Ed. Engl. 52, 1494 (2013).

[61] F. Hong, B. Yue, N. Hirao, Z. Liu, and B. Chen, Significant Improvement in $\mathrm{Mn}_{2} \mathrm{O}_{3}$ Transition Metal Oxide Electrical Conductivity via High Pressure, Sci. Rep. 7, 44078 (2017).

[62] S.-H. Shim, T. S. Duffy, R. Jeanloz, C.-S. Yoo, and V. Iota, Raman Spectroscopy and X-ray Diffraction of Phase Transitions in $\mathrm{Cr}_{2} \mathrm{O}_{3}$ to 61 GPa, Phys. Rev. B 69, 144107 (2004).

[63] S. V. Ovsyannikov, X. Wu, G. Garbarino, M. NúñezRegueiro, V. V. Shchennikov, J. A. Khmeleva, A. E. Karkin, N. Dubrovinskaia, and L. Dubrovinsky, High-Pressure Behavior of Structural, Optical, and Electronic Transport Properties of the Golden $\mathrm{Th}_{2} \mathrm{~S}_{3}$-Type $\mathrm{Ti}_{2} \mathrm{O}_{3}$, Phys. Rev. B 88, 184106 (2013).

[64] V. Potapkin et al., Effect of Iron Oxidation State on the Electrical Conductivity of the Earth's Lower Mantle, Nat. Commun. 4, 1427 (2013).

[65] Z. Mao, J. Lin, J. Yang, T. Inoue, and V. B. Prakapenka, Effects of the $\mathrm{Fe}^{3+}$ Spin Transition on the Equation of State of Bridgmanite, Geophys. Res. Lett. 42, 4335 (2015). 